\title{
Method of Construction of Material That Work on All the Range of Wavelengths or Frequency or Energy of Photon
}

\author{
Ram Chandra Pageni, Saddam Husain Dhobi, Narayan Panthi, Sonam Gyaljen Tamang, \\ Sudarshan Shrestha \\ Department of Physics, Tribhuvan University, Kathmandu, Nepal \\ Email: pagenirc09@gmail.com, saddamdhobe@gmail.com,narayan.panthi321@gmail.com, gyaljen511@gmail.com, \\ sudson292017@gmail.com
}

How to cite this paper: Pageni, R.C., Dhobi, S.H., Panthi, N., Tamang, S.G. and Shrestha, S. (2019) Method of Construction of Material That Work on All the Range of Wavelengths or Frequency or Energy of Photon. Journal of Applied Mathematics and Physics, 7, 1826-1839.

https://doi.org/10.4236/jamp.2019.78125

Received: July 4, 2019

Accepted: August 16, 2019

Published: August 19, 2019

Copyright $\odot 2019$ by author(s) and Scientific Research Publishing Inc. This work is licensed under the Creative Commons Attribution International License (CC BY 4.0).

http://creativecommons.org/licenses/by/4.0/

\begin{abstract}
The main objective of this research work is to decrease work function of any given element or compound or material. To decrease the work function of the given material we have to decrease the bandwidth between conduct band and valance band. Because according to definition of work function, the amount of energy that required the remove the electron from valance band of an atom and it is also called ionization energy. These all energies depend upon the band width that is greater than the band width greater energy required to remove the electron from the surface, and less than the band width and lesser amount of energy required to remove the electron from of materials. In this work we are trying to give an theoretical model or relation, how to decrease the work function of a material by applying external pressure on atoms and doping of the material that has screening or shielding effects. With the help of this model we can increase the efficiency of material used in solar cell that is cell work for all range of frequencies and by construction material bases on this we can increase the efficiency of solar cell or any type of material working solar cell principle.
\end{abstract}

\section{Keywords}

Conduction Band, Ionization Energy, Solar Cell, Valance Band, Work Function

\section{Introduction}

The total amount of energy received at ground level from the Sun is about 3.3\% higher than average in January and lower in July. In terms of energy, sunlight at 
Earth's surface is around 52 to 55 percent infrared 42 to 43 percent visible, and 3 to 5 percent ultraviolet. In this research work, we are trying to give a theoretical model, that decreases the work function of the material especially, photonic material from IR to Visible range frequencies because the maximum amount of energy in our atmosphere is energy of photon. The utilization of such huge amount of energy is possible only if we constructed materials which can emit the electron from atom, for all ranges of frequencies. This is possible to decrease the work function of the material either by doping of the different material that has shielding effect or by applying the Van Der Waals force relation. Base on these principles we can construct photonic materials that have different work function and can be used for different frequency of photon especially IR and Visible photon.

Photonic crystals are periodically structured electromagnetic media, generally possessing photonic band gaps: ranges of frequency in which light cannot propagate through the structure. The study of photonic crystals is likewise governed by the Bloch F. theorem, and intentionally introduced defects in the crystal. Felix Bloch pioneered the study of wave propagation in three dimension-ally periodic media in 1928, which proved that waves in such a medium could propagate without scattering, when the photon incident on the material the energy of the photon observed electron or electron cloud of an atom. The energy observation is started from the outer most valance orbital i.e. the outer most electron of an atom and goes on the excited state or kicks out from the orbits of an atom; hence the photoelectric effect take place. Let the energy of incident photon on the surface of photonic material is $h f$, where $h$ is plank constant and $f$ is frequency and the amount of energy required to remove or electron from its valance orbit to conduct orbit $h f_{1}$ where $f_{1}$ is frequency needed to excite the electron from valance band or orbit1 and the energy $h f_{2}$ of excited state, where $f_{2}$ is frequency of exciting the electron for conducing band or orbit 2 and the difference between them gives, $h f=h f_{2}-h f_{1}$, which implies smaller the difference of $h f_{2}-h f_{1}$, where $h f$ is smaller that is $f$ is smaller and shows that on changing the energy level or orbit difference we can obtain the necessary value of $h f_{2}-h f_{1}$. In this way we can change frequency for our desire and increase the efficiency of the photonic materials like solar cell.

On the other hand, work function of the material is define as the minimum amount of the energy required to remove the electron from the material surface which is given by $h f_{3}$ where $h=$ plank's factor and $f_{3}$ is the minimum frequency required to remove the electron from the surface, which is equal to or less than incident frequency; then we can obtain the relation.

$$
h f_{2}-h f_{1}=h f_{3} \leq h f
$$

In general,

$$
h f_{n+1}-h f_{n}=h f_{n^{\prime}} \leq h f
$$

In Equation (2), $f_{n+1}$ is frequency of orbit $n+1$ and $f_{n}$ is the frequency of orbit 
$n$.

Now on putting the value of $f=\frac{c}{\lambda}$ where $c=$ velocity of light and $\lambda$ in Equation (2) we obtain the relation

$$
1 / \lambda_{n+1}-1 / \lambda_{n}=1 / \lambda_{n^{\prime}}=1 / \lambda
$$

where $\lambda_{n+1}, \lambda_{n}$ and $\lambda_{n}$, or $\lambda$ is corresponding wavelength of $n+1, n$ orbit and difference wavelength of two orbit $n+1$ and $n$.

Photonic crystals usually consist of dielectric materials, that is, materials that serve as electrical insulators or in which an electromagnetic field can be propagated with low loss. Materials used for making a Photonic band gap are: Silicon, Germanium, Gallium and so on.

\section{Literature}

Composite optical materials can display useful optical properties that are qualitatively dissimilar from those of their underlying constituents. Nano composite materials are especially well suited for photonic applications because they can be constructed in such a manner as to produce enhanced nonlinear optical response. More recently entangled states of light have been used to perform functions unthinkable in the context of classical physics, such as the demonstration of quantum cryptography and quantum teleportation (those materials which interact with certain frequency that is we can transform quantum information from one to another place). One application entails the development of techniques for the construction of imaging systems that can achieve a transverse resolution that exceeds the classical Rayleigh criterion [1]. Photonic crystal working in the optical range of electromagnetic spectrum but PCs for X-ray should present a modulation of some $\mathrm{A}^{0}$ which is a solid state crystal. EM field store more energy if it has extreme at the region of high dielectric function. Bragg diffraction is the primary feature of the system of PBG material can be used for initial characterization. When refractive index contrast is low it is good approx. to use ordinary diffraction or dynamic to characterized PCs [2]. The mid-infrared wavelength range from 2 to 20 microns is a spectral region of tremendous interest which is important for a wide range of applications ranging from chemical and bio-Sensing to spectroscopy and thermal imaging, optical fiber can transmit the light from visible to near IR, industrial and military applications, such as remote sensing and explosive detection or free-space communication systems [3].

The electromagnetic band gap overlaps the electronic band edge by at least a few $\mathrm{kT}$ in energy, then electron-hole radioactive recombination, which creates periodic three-dimensional dielectric structures in which there exists an electromagnetic band gap. The recombination rate of electrons and holes in a semiconductor can be expressed as a power series in the injected carrier density. The combined electron and hole three-body Auger recombination coefficient are intrinsic to the material. In today's high quality, double hetero structure has low 
enough defect density [4]. The concept of a photonic material is explained in terms of Bragg diffraction. The concept of Bragg reflection applies equally well to visible radiation except that we cannot rely on atoms to do work for all researcher [5]. GaAs are used in various electronic applications such as wireless communication, microwave, and high-speed digital systems. Silicon appears as thin films of amorphous or poly crystalline form in TFT-technology, in solar cell. $\mathrm{SiGe}$ is needed to introduce electron accelerating strain in the Si lattice. GaAs, InP, and GaP, has occurred culminating in the development of a blue semiconductor laser using $\mathrm{GaN}$ [6]. As in the 3D case, we can create dielectric system which exhibits a complete photonic band gap, a set of frequencies in which light of any polarization cannot propagate in any in-plane direction and drill air columns in dielectric. At the dielectric contrast of GaAs, the only combination which was found to have a photonic band gap in both polarization was the triangular lattice of air columns in dielectric [7]. Photonic crystals can be used to selectively reflect specific frequencies of electromagnetic radiation. It has recently been shown experimentally that geometrically asymmetric silicon structures support strong near-infrared magnetic resonances akin to the familiar 'trapped mode' of metallic asymmetric split ring designs. Silicon and silicon nit-ride are assumed lossless with refractive indices of 3.5 and 2.0 respectively in the near-infrared range under consideration. All in-plane optical forces generated within the meta-material structure are canceled and only out-of plane forces act on the dielectric beams. These drive each beam to move up or down until elastic restoring forces balance the optical forces. Optical absorption and variations in ambient temperature may lead to thermo-mechanical changes in the structure [8] [9].

Far-infrared radiation traveling through a polar crystal, such as GaAs, couples strongly to optical phonon. The transverse optical (TO) and longitudinal optical (LO) phonon define a region of negative permittivity and the center of a region of high loss, the so-called "Reststrahlen band" of the material. Optical transparency in the $20-60 \mu \mathrm{m}$ range for intrinsic III-V semiconductors is limited by absorption due to optical phonon. Consequently, materials and devices based on III-V semiconductors are scarce in the Reststrahlen region [10]. Beyond semiconductors, superconductors also have the potential to advance the state-of the-art in far-IR detection. Microwave kinetic inductance detectors sense incident radiation by detecting small changes in the inductance of a thin superconductor from the breaking of Cooper pairs. The small changes in the inductance can be detected by monitoring the resonance frequency of a microwave resonator that incorporates the superconducting film. A challenge associated with $\mathrm{Mi}$ crowave kinetic inductance detectors for the far-IR is coupling incident radiation into the device [11].

A well-known thin-film Gallium nit-ride LED structure is fabricated by removing the sapphire layer using a laser and roughening the revealed n-doped Gallium nit-ride layer. Gallium nit-ride thin-films, wafer-bonded to oxidized 
silicon wafers, provide a promising platform for nonlinear integrated optics. The obtainable quality factors in optical micro-resonators based on bonded Gallium nit-ride thin films are limited by the remaining surface roughness. Aluminum nit-ride, on the other hand, is a wide-band gap semiconductor with the capability of direct integration on silicon (100) substrates with suitable mechanical and thermal properties [12] [13].

Photonic crystals have a periodic dielectric structure with high index contrast, designed to control photons in the same way that conventional crystals control electrons. PCs also possess a band gap, a so-called photonic band gap, where the material acts like a photonic insulator and light of certain frequencies cannot propagate. The Electro optics effect is the change in the index of refraction of a material with an applied external electric field, given by $\delta$ - $n_{i j}=-n_{i j}^{2}\left(r_{i j k} E_{k}+s_{i j k l} E_{k} E_{l}\right)$ [14]. Transparent electrodes are used to apply an electric field, which, in combination with polarized, may be used to either block or transmit the light. Electro optic crystals have extremely small Electro optic coefficients. Liquid crystal optical-phased array beam steers tend to be slow, provide non-continuous diffraction steering, and have a very limited steering range because thick LC layers are problematic. Acoustic optic beam steers have a larger steering range but are also diffraction, require very large power supplies and expensive crystals [15].

Photonic crystals are meta-materials designed to display a periodic modulation of the refractive index. One of the intrinsic shortcomings of photonic crystals is the highly selective reflection from Bragg planes due to crystalline symmetries. For example, dye-free reflective color displays, colored packing materials or cosmetics are preferentially non-iridescent and thus non-crystalline. For a photonic crystal of thickness $L$ the sum of transmittance $T$ and spectacular reflectance $R$ is $T+R=1$. The gap wavelength $\lambda_{G}$ and the transmittance displays an exponential decay $T=\mathrm{e}^{-L / L_{B}}$, where $L_{B}$ is the Bragg length [16].

AlGaN alloys have been the default choice for the development of Deep ultraviolet Op-to-electronic devices. Deep ultraviolet light emitting diodes LED and laser diodes with high quantum efficiencies QEs is the low conductivity of p-type $\mathrm{AlGaN}$. The resistivity of Mg-doped AlGaN increases with Al-content and becomes extremely high in Mg-doped AlN. Seebeck effect measurement is a well-established technique to distinguish between n-type and p-type conductivity of a semiconductor. The temperature gradient creates a voltage between the cold and hot ends due to the diffusion of thermally excited charged carriers [17].

As the investigation of photo current generation mechanisms in $2 \mathrm{D}$ opt o electronics goes deeper, 2D material-based devices have also been demonstrated to exhibit elevated performance. Over the past decade, it has been shown that most graphene based photo-transistors exhibit photo responsive around 10 $\mathrm{mA} / \mathrm{W}$ [18]. First reported single-layer $\mathrm{MoS}_{2}$ photo transistors reach responsibility to $7.5 \mathrm{~mA} / \mathrm{W}$ with $50 \mathrm{~V}$ gate bias, and exhibit stable response time within $50 \mathrm{~ms}$. Because mono layer $\mathrm{MoS}_{2}$ is a direct-band gap semiconductor due to 
quantum-mechanical confinement, it could be suitable for applications in opt o electronic devices where the direct band gap would allow a high absorption coefficient and efficient electron-hole pair generation under photo excitation. The maximum external photo is responsive of $880 \mathrm{~A} \mathrm{~W}-1$ at a wavelength of $561 \mathrm{~nm}$ and a photo response in the $400-680 \mathrm{~nm}$ range. $\mathrm{MoS}_{2}$ shows important potential for applications in $\mathrm{MoS}_{2}$-based integrated opt o electronic circuits, light sensing, biomedical imaging, video recording and spectroscopy [19] [20].

Near the gap in a photonic band, gap material the effective index of refraction can become less than unity and in fact can approach zero at the band edge itself-leading to ultra-refractive optical effects. Ultra-refractive optics with photonic band materials has many applications, including laser accelerators and lenses of ultra-short focal lengths. Scully and colleagues, In current prototypes of PBG materials the structure is about $85 \%$ air and so losses are less than in a homogeneous dielectric and discussed the novel properties of the effective index of refraction of PBG material when operating near a band edge, using a quantitative and simple one-dimensional model of a three-dimensional PPG structure [21].

We present designs of 2D, isotropic, disordered, photonic materials of arbitrary size with complete band gaps blocking all directions and polarization. The designs with the largest band gaps are obtained by a constrained optimization method that starts from a hyper uniform disordered point pattern Since their introduction in 1987, photonic band gap (PBG) materials have evolved dramatically, and their unusual properties have led to diverse applications. Obtaining complete PBGs in dielectric materials without long-range order is counter intuitive. If the arrangement of dielectrics has local geometric order, a tight binding model with nearly uniform coefficients describes the propagation of light in the limit of high dielectric constant ratio. Weaire and Thorpe proved that band gaps could exist in continuous random tetrahedral coordinated networks, commonly used as models for amorphous silicon and germanium. The comparison to electronic band gaps is also useful in comparing states near the band edges and continuum. For a perfectly ordered crystal (or photonic crystal), the electronic (photonic) states at the band edge are propagating such that the electrons (electromagnetic fields) sample many sites. If modest disorder is introduced, localized states begin to fill in the gap so that the states just below and just above are localized. Although formally the disordered hetero-structures do not have equivalent propagating states, an analogous phenomenon occurs [22].

The radiation effects on Ge-doped and (Fluorine) F-doped fibers and performs: the first play a crucial role in the photo sensitivity property, the second improves the dielectric radiation hardness even at low concentrations. Usually classical optical fibers for telecommunications are used in the IR, from 835 to $1600 \mathrm{~nm}$, medical application and plasma diagnostic use of visible and UV region were optical transmission is affected by many losses. Fluorine is a promising key material in optical fiber technology directed to applications requiring 
high and stable transmission in UV and visible spectral range [23]. Photons interact very weakly with transparent optical media. Due to their fast propagation speed, photons use for transmitting quantum information and distributing quantum correlations. Additionally, photons possess many degrees of freedom; include spatial and temporal modes, frequency, polarization and angular momentum. Photons also possess continuous quantum variables, such as the quantized field quadrature, which can be utilized in special classes of information protocols. Silicon has a much higher refractive index than silica, leading to 1000 times smaller wave guide bend radius compared to that in silica wave guides. Silicon's indirect band gap of $1.12 \mathrm{eV}$ and low intrinsic carrier concentration makes it transparent to photons at the telecommunication wavelength $(1.55 \mu \mathrm{m})$ [24].

Up to now, entangled photon pairs have been generated by optical pumping in passive semiconductor wave guides by exploiting four-wave mixing in silicon or spontaneous parametric down conversion in aluminum gallium arsenide. Aluminum Gallium Arsenide device that emits photon pairs at telecom wavelengths and operates at room temperature [25]. Three-dimensional confinement of both electrons and holes in hetero-structures gives rise to quantum dots which potentially provide on-demand single-photon generation across the NIR range with near unity internal efficiency. Efficient photon collection represents a major technological challenge associated with quantum emitters. Integrated collection techniques with over $98 \%$ mode coupling efficiency exist for quantum dots [26].

InGaAs/GaAs quantum dots demonstrate single-photon emission for wavelengths up to $1400 \mathrm{~nm}$, while InAsP/InP quantum dots emission can be achieved across the entire telecom spectrum. Multi-photon entangled states can be generated, constituting a particularly useful resource for quantum communication. The combination of a diode junction and an embedded quantum dot has led to the demonstration of an electrically driven entangled-photon source of high enough quality to perform quantum teleportation [27] [28].

It is also found that the size and composition with alloy effect can tune the band-gap energy, which suggests an effective way to reach the desirable electrical and optical properties. Interestingly, both the size and the composition can tune the band-gap energy of Nano compounds. In particular, the composition tun-able band-gap may be a better way for wider band-gap materials [29]. Ionization potential is the electric potential $(\mathrm{V})$ required to separate an electron from the orbital system in free space with the kinetic energy remaining unchanged. Ionization energy is the work done in removing the electron at zero temperature and is measured conveniently in electron volts, where $1 \mathrm{eV}=1.6022 \times 10^{19} \mathrm{~J}$. Screening (electron-electron repulsion) reduces electron-nucleus attractions in helium and two-electron atomic ions but ionization energies are not functions of simple squares. First ionization energies vary in the order $\mathrm{B}>\mathrm{Al}<\mathrm{Ga}>\mathrm{In}<\mathrm{Tl}$ and $\mathrm{C}>$ $\mathrm{Si}>\mathrm{Ge}>\mathrm{Sn}<\mathrm{Pb}$ but decrease with increasing atomic number down groups 15 
to 18 . In 1930 , Slater give a relation $I=R\left(Z^{*} / N^{*}\right)^{2}$, where $Z=$ an effective nuclear charge, $N^{*}=$ an effective quantum number could be assigned to each electron and $I=$ energy required to ionize an electron [30].

The photo-ionization of $3 \mathrm{~d}^{\prime \prime}$ impurities in semiconductors with zinc blende structure is to be treated by investigating the matrix elements for electric dipole transitions between linear combinations of d-type one-electron wave functions which can be mixed with p-states and the continuum of states in the conduction band, i.e. Bloch functions [31]. The force attracting the electron to the nucleus depends upon magnitude of the core charge, and the separation of the electron from the nucleus. The first ionization energy will therefore be the work done when removing an electron from the core and so will be that needed to pull the electron away against the attraction from the core charge. This will not be an infinite quantity as the magnitude of the force falls rapidly with distance [32].

The ionization energy (IE) is defined as the minimum amount of energy that needs to be absorbed by an atom or molecule in its electronic and vibration ground states in order to form an ion that is also in its ground states by ejection of an electron. Ionization energies of most molecules are in the range of $7-15$ $\mathrm{eV}$. The ionization cross section describes an area through which the electron must travel in order to effectively interact with the neutral [33]. Lebedev gave the radiation pressure of light in 1901. In principle, if the currents in the mirror/antenna can be determined from the drive fields, then the electromagnetic fields due those currents can be calculated, and combined with the drive fields to obtain the total fields. This effect of the currents on themselves can be expressed mathematically as an integral equation for the currents, which takes into account the good-conductor boundary condition at the surface of the mirror/antenna [34].

\section{Methodology: Theoretical Calculation}

In this paper we are trying to give theoretical model or relation that help to construct such type of material which work on difference range of frequencies that is produce the current or energy for all frequencies. To give the theoretical relation here we follow the Bohr's relation of energy for hydrogen atom and pressure area relation.

If we increase the pressure on an atom surface externally, we can decrease the width or the distance between two outer most orbits of an atom that is the two outer most orbits come closure. This shows amount of energy is required to excite electron from inner outer most orbit to outer most orbit needed less. But in normal condition or without applying the pressure on atom the distance between the inner outer most and outer most is quite greater then applied presure condition and hence the energy required to excite the electron from inner most outer orbit to outer most orbit needed more.

Here the outer most orbit is last orbit of an atom when we consider the counting of orbit from nucleus and the inner outer most orbit is second last orbit of nucleus. 


\section{Mathematical Derivation:}

From hydrogen model we have Bohr's energy for different orbits. Let us consider the energy of valance band or energy of ground state electron is $E_{n}$ and conductance band or excited state electron is $E_{n+1}$.

Now we relation $E_{n+1}-E_{n}=h f$ when an electron goes from ground state to excited state and on coming to ground state again by emitting the energy of $h f$.

Similar phenomena we are using here that is amount of energy required to go electron from valance band to conduct band is given by $E_{n+1}-E_{n}=h f$, where $h$ $=$ planks factor and $f=$ incident frequency of a photon. Let us consider, $N=$ Nucleus of an atom, $n=1$ is the nearest orbit of nucleus where electron is bounded tightly, $n=2$ is orbit of nucleus where electron is less bounded then $n$ $=1, n=3$ is another orbit where electron is less bounded then $n=2$. In similar way the electron bounded is going decrease as we go far away from nucleus. As shown in Figure 1.

According to Bohr's the amount of energy required to bounded the electron around the nucleus is given by

$$
E_{n}=-\frac{1}{n^{2}} \frac{m e^{4} z^{2}}{8 \varepsilon^{2} h^{2}}=-\frac{z^{2} R_{H}}{n^{2}}
$$

where $R_{H}=$ Rydberg's constant.

We also have, Bohr's radius

$$
r_{n}=\frac{n^{2} h^{2}}{z 4 \pi^{2} m e^{2}}=0.529 \times \frac{n^{2}}{z}
$$

This is the distance from the center of nucleus to the orbit of an electron revolving around the nucleus.

$$
\text { Or, } \frac{1}{n^{2}}=\frac{0.529}{z r_{n}}
$$



Figure 1. Energy Level for an atom without applying the external pressure. 
Now From Equations (4) and (5) we have

$$
E_{n}=-\frac{0.529 z R_{H}}{r_{n}}
$$

This relation shows that how much amount of energy required to bind the electron by proton or nucleus when the electron is at a certain i.e. $r_{n}$.

Let us consider, $E_{n+1}$ is the amount of energy required to remove the electron from the outer shell of atom of any atom is at a distance $r_{n+1}$ then the energy can be obtain similar as Equation (6).

Since we have an energy relation

$$
E_{n+1}=-\frac{0.529 z R_{H}}{r_{1+n}}
$$

In other word, $E_{n+1}$ and $E_{n}$ is the energy level of valance band or ground band of orbit $n$ and $n+1$ of an atom then the transition between them is possible. If we consider $n$ as ground state and $n+1$ is excited state of electron in an atom then, Now on subtracting Equation (7) from (6) we have,

$$
h f=E_{n+1}-E_{n}=0.529 \mathrm{z} R_{H}\left(\frac{1}{r_{n}}-\frac{1}{r_{n+1}}\right)
$$

where $h f$ is incident photon, which is needed to, excited an electron from one energy level to another that means from $E_{n}$ to $E_{n+1}$ level.

$$
h f=E_{n+1}-E_{n}=0.529 z R_{H}\left(\frac{r_{n+1}-r_{n}}{r_{n} \times r_{n+1}}\right)
$$

Here from Equation (9) we have an interesting relation that the different between two energy levels are related to the distance between two orbits is directly proportional and product is inversely related which means incident photon energy is directly related difference of $r_{n+1}-r_{n}$ it means that smaller the difference smaller the amount of energy required to excited an electron from $r_{n}$ to $r_{n+1}$. And another relation is inversely related to the product of the distance of two orbits from the nucleus $\left(r_{n} \square r_{n+1}\right)$.

Since the atom is not solid that is within atom there is the space so we can compress the atom. Here we are studying the compression of the atom from the outer surface area during the compression the of an atom equal from all sides. the outer shell come to closure to the second last orbit or ground state orbit while the ground state orbit is less effect due to apply the pressure from outer side of atom.

Lets $P_{n}$ and $P_{n+1}$ are the pressure applying on energy level of an atom which is at a distance of $r_{n}$ and $r_{n+1}$ from nucleus, with constant force $F$ then pressure exerted on $n$ and $n+1$ orbits.

Then external pressure is applied on orbit of $n+1$ and $n$ orbit with constant force to an atom is given by

$$
P_{n}=\frac{F}{A_{n}}=-\frac{F}{4 \pi r_{n}^{2}}
$$




$$
P_{n+1}=\frac{F}{A_{n+1}}=-\frac{F}{4 \pi r_{n+1}^{2}}
$$

respectively. Here negative sign in (10) and (11) implies show that force is acting from outside that mean in outer most orbit. Since the pressure is applied from outside or surface of an atom then pressure exerted on $n+1$ orbit is greater then pressure exerted on $\mathrm{n}$ orbit for constant force.

Now on subtracting Equation (10) from (11) we have,

$$
\begin{gathered}
P_{n+1}-P_{n}=\frac{F}{4 \pi}\left(\frac{1}{r_{n}^{2}}-\frac{1}{r_{n+1}^{2}}\right) \\
P_{n+1}-P_{n}=\frac{F}{4 \pi}\left(\frac{r_{n+1}^{2}-r_{n}^{2}}{r_{n}^{2} \times r_{n+1}^{2}}\right)
\end{gathered}
$$

Now, from Equation (13) we have clearly, see that pressure different is directly related to different and inversely related to square product of distance from nucleus to electron.

$$
P_{n+1}-P_{n}=\frac{F}{4 \pi}\left(\frac{\left(r_{n+1}-r_{n}\right)\left(r_{n+1}+r_{n}\right)}{r_{n}^{2} \times r_{n+1}^{2}}\right)
$$

Now, on putting the value from Equation (9) on Equation (14), we have,

$$
P_{n+1}-P_{n}=\frac{F}{4 \pi}\left(\frac{\left(E_{n+1}-E_{n}\right)\left(r_{n+1}+r_{n}\right)}{0.529 z R_{H}\left(r_{n+1} \times r_{n}\right)}\right)
$$

Since $P_{n+1}$ is greater than $P_{n}$ then $P_{n+1}-P_{n}$ must be positive which is also depend up on the $\left(E_{n+1}-E_{n}\right)$ of Equation (15) which is depend upon $\left(r_{n+1}-r_{n}\right)$.

It is possible because of the atom has space according to the Ruth-er forth scattering of an atom. Here we can press the electron for the certain radius because of repulsion of nuclear. In addition, Van Der Waals forces between atoms are depends upon the sized of atoms that is smaller the sized smaller the Van Der Waals force. Also on doping the material having the greater atomic number decrease attraction of the electron with the nucleus that is less amount of energy required to remove the electron from the surface of atom or materials. From the above Methodology, we can decrease the work function of the element or compound or materials.

\section{Result and Conclusion}

Hence, from the above relation (15), pressure, energy and orbit radius we can change the work function of material and make them work for all frequencies ranges. This helps to increase the efficiency of solar cell material or similar principle material. Once material is constructed or follows this principle, energy crisis should be ended forever. In other hand, we can also say that, same materials can be used for different range of frequencies and extracted energy from all ranges radiation. Hence such material can also solve the problem of energy where solar radiation is absent. 


\section{Acknowledgements}

We would like to thanks all the members of Innovative Ghar Nepal and Robotic Academy Nepal who directly and indirectly provide us research space and support during our research work. Similarly, we would like to thanks our family member who provide peaceful environment during my research work.

\section{Conflicts of Interest}

The authors declare no conflicts of interest regarding the publication of this paper.

\section{References}

[1] Boyd, R.W. (2003) Novel Photonic Materials for Advanced Imaging Applications. Journal of the Korean Physical Society, 43, 603-605.

https://www.researchgate.net/publication/228415943_Novel_Photonic_Materials_fo r_Advanced_Imaging_Applications

[2] Lopez, C. (2003) Material Aspect for Photoinc Crystal. Advance Material, 15, 1679-1684. https://onlinelibrary.wiley.com/doi/abs/10.1002/adma.200300386 https://doi.org/10.1002/adma.200300386

[3] Academy of Finland Research Programmes (2013) Foresight: Materials for Photonics.

https://www.aka.fi/globalassets/32akatemiaohjelmat/oma/fotoniikka_ennakointiesit e.pdf

[4] Yablonovitch, E. (1987) Inhibited Spontaneous Emission in Solid-State Physics and Electronics. Physical Review Letters, 58, 2059-2061.

https://www.iap.uni-jena.de/iapmedia/de/Lecture/Nanomaterials1367272800/Nano

Mat12_Paper_08.pdf

https://doi.org/10.1103/PhysRevLett.58.2059

[5] Pendry, J.B. (1999) Photonic Gap Materials. Current Science, 76, 1311-1136.

[6] Hoff, A.M. and Ruzyllo, J. (2006) Electronics and Photonics. In: The Electrochemical Society Interface, Springer, Berlin, 36-37.

[7] Meade, R.D., Brommer, K.D., Rappe, A.M., and Joannopoulos, J.D. (1992) Existence of a Photonic Band Gap in Two Dimensions. Applied Physics Letter, 61, 495-496. https://aip.scitation.org/doi/10.1063/1.107868

https://doi.org/10.1063/1.107868

[8] Pouya, C., Overvelde, J.T.B., Kolle, M., Aizenberg, J., Bertoldi, K., Weaver, J.C. and Vukusic, P. (2015) Characterization of a Mechanically Tunable Gyroid Photonic Crystal Inspired by the Butterfly Parides sesostris. Advanced Optical Materials, 4, 99-105. https://doi.org/10.1002/adom.201500436

[9] Zhang, J., MacDonald, K.F. and Zheludev, N.I. (2013) Nonlinear Dielectric Optomechanical Metamaterials. Light. Science \& Applications, 2, e96. https://doi.org/10.1038/lsa.2013.52

[10] Feng, K., Streyer, W., Zhong, Y., Hoffman, A.J. and Wasserman, D. (2015) Photonic Materials, Structures and Devices for Reststrahlen Optics. Optics Express, 23, A1418. https://doi.org/10.1364/OE.23.0A1418

[11] Stevenson, T.R., Adams, J.S., Hsieh, W., Moseley, S.H., Travers, D.E., Uyen, K., Wollack, E.J. and Zmuidzinas, J. (2009) Superconducting Films for Absorber-Coupled 
MKID Detectors for Sub-Millimeter and Far-Infrared Astronomy. IEEE Transactions on Applied Superconductivity, 19, 561-564. https://doi.org/10.1109/TASC.2009.2019661

[12] Huang, H.W., Lin, C.H., Huang, Z.K., Lee, K.Y., Yu, C.C. and Kuo, H.C. (2010) Double Photonic Quasi-Crystal Structure Effect on GaN-Based Vertical-Injection Light-Emitting Diodes. Japanese Journal of Applied Physics, 49, Article ID: 022101. https://pdfs.semanticscholar.org/1b32/13ee213c10244da09e2cd1993270768a2bf6.pdf https://doi.org/10.1143/JJAP.49.022101

[13] Pernice, W.H.P., Xiong, C., Schuck, C. and Tang, H.X. (2012) High-q Aluminum Nitride Photonic Crystal Nanobeam Cavities. Applied Physics Letters, 100, Article ID: 091105. https://doi.org/10.1063/1.3690888

[14] Scrymgeour, D., Malkova, N., Kim, S. and Gopalan, V. (2003) Electro-Optic Control of the Superprism Effect in Photonic Crystals. Applied Physics Letters, 82, 3176-3178. https://aip.scitation.org/doi/10.1063/1.1574402 https://doi.org/10.1063/1.1574402

[15] Davis, S.R., Rommel, S.D., Farca, G. and Anderson, M.H. (2008) A New Generation of Previously Unrealizable Photonic Devices as Enabled by a Unique Electro-Optic Waveguide Architecture. SPIE, Vol. 7050, 8-9.

[16] Muller, N., Haberko, J., Marichy, C. and Scheffold, F. (2013) Silicon Hyper Uniform Disordered Photonic Materials with a Pronounced Gap in the Shortwave Infrared. Advanced Optical Materials, 2, 115-119. https://doi.org/10.1002/adom.201300415

[17] Dahal, R., Li, J., Majety, S., Pantha, B.N., Cao, X.K., Lin, J.Y. and Jiang, H.X. (2011) Epitaxially Grown Semiconducting Hexagonal Boron Nitride as a Deep Ultraviolet Photonic Material. Applied Physics Letters, 98, Article ID: 211110.

https://aip.scitation.org/doi/10.1063/1.3593958 https://doi.org/10.1063/1.3593958

[18] Xia, F., Mueller, T., Lin, Y.M., Garcia, A.V. and Avouris, P. (2009) Ultrafast Graphene Photodetector. Nature Nanotechnology, 4, 839-843.

http://www.gr-sci.net/papers/nnano.2009.292.pdf https://doi.org/10.1038/nnano.2009.292

[19] Yin, Z., Li, H., Li, H., Jiang, L., Shi, Y., Sun, Y., Lu, G., Zhang, Q., Chen, X. and Zhang, H. (2011) Single-Layer $\mathrm{MoS}_{2}$ Phototransistors. ACS Nano, 6, 74-80.

https://pubs.acs.org/doi/10.1021/nn2024557 https://doi.org/10.1021/nn2024557

[20] Sanchez, O.L., Lembke, D., Kayci, M., Radenovic, A. and Kis, A. (2013) Ultrasensitive Photodetectors Based on Monolayer $\mathrm{MoS}_{2}$. Nature Nanotechonology, 8, 497-499. https://www.nature.com/articles/nnano.2013.100 https://doi.org/10.1038/nnano.2013.100

[21] Dowling, J.P. and Bowden, C.M. (1994) Anomalous Index of Refraction in Photonic Bandgap Materials. Journal of Modern Optics, 41, 345-351.

https://doi.org/10.1080/09500349414550371 https://www.tandfonline.com/doi/abs/10.1080/09500349414550371

[22] Florescu, M., Torquato, S. and Steinhardt, P.J. (2009) Designer Disordered Materials with Large. Complete Photonic Band Gaps. Proceedings of the National Academy of Sciences Proceedings of the National Academy of Sciences, 106, 20658-20663. https://doi.org/10.1073/pnas.0907744106

[23] Origlio, G., Girard, S., Boscaino, R., Boukenter, A., Cannas, M. and Ouerdane, Y. (2009) Optical and Photonic Material Hardness for Energetic Environments. UVX, 2008, 127-132. https://doi.org/10.1051/uvx/2009020 
[24] Bogdanov, S., Shalaginov, M.Y., Boltasseva, A. and Shalaev, V.M. (2017) Material Platforms for Integrated Quantum Photonics. Optical Materials Express, 7, 111-132. https://arxiv.org/pdf/1610.00729

[25] Boitier, F., Orieux, A., Autebert, C., Lemaître, A., Galopin, E., Manquest, C., Sirtori, C., Favero, I., Leo, G. and Ducci, S. (2014) Electrically Injected Photon-Pair Source at Room Temperature. Physical Review Letters, 112, Article ID: 183901. https://link.aps.org/doi/10.1103/PhysRevLett.112.183901 https://doi.org/10.1103/PhysRevLett.112.183901

[26] Arcari, M., Söllner, I., Javadi, A., Hansen, S.L., Mahmoodian, S., Liu, J., Thyrrestrup, H., Lee, E.H., Song, J.D., Stobbe, S. and Lodahl, P. (2014) Near-Unity Coupling Efficiency of a Quantum Emitter to a Photonic Crystal Waveguide. Physical Review Letters, 113, Article ID: 093603.

https://link.aps.org/doi/10.1103/PhysRevLett.113.093603

https://doi.org/10.1103/PhysRevLett.113.093603

Salter, C.L., Stevenson, R.M., Farrer, I., Nicoll, C.A., Ritchie, D.A. and Shields, A.J. (2010) An Entangled-Light Emitting Diode. Nature, 465, 594-597.

https://www.researchgate.net/publication/44646833_An_entangled_light-emitting diode https://doi.org/10.1038/nature09078

[27] Nilsson, J., Stevenson, R.M., Chan, K.H.A., Szymanska, J.S., Lucamarini, M., Ward, M.B., Bennett, A.J., Salter, C.L., Farrer, I., Ritchie, D.A. and Shields, A.J. (2013) Quantum Teleportation Using a Light Emitting Diode. Nature Photonics, 7, 311-315. https://www.nature.com/articles/nphoton.2013.10?draft=journal https://doi.org/10.1038/nphoton.2013.10

[28] Wang, Y., Ouyang, G., Wang, L.L., Tang, L.M., Tang, D.S. and Sun, C.Q. (2008) Size and Composition Induced Band-Gap Change of Nano Structured Compound of II-VI Semiconductors. Chemical Physics Letters, 463, 383-386. https://www3.ntu.edu.sg/home/ecqsun/RTF/CPL_WangY.pdf https://doi.org/10.1016/j.cplett.2008.08.083

[29] Lang, P.F. and Smith, B.C. (2003) Ionization Energies of Atoms and Atomic Ions. Journal of Chemical Education, 80, 938-946.

https://pubs.acs.org/doi/abs/10.1021/ed080p938

https://doi.org/10.1021/ed080p938

[30] Grebe, G. and Schulz, H.J. (2013) Luminescence of $\mathrm{Cr}^{2+}$ Centres and Related Optical Interactions Involving Crystal Field Levels of Chromium Ions in Zinc Sulfide. Verlag Zeitschrift für Naturforschung, 29, 1808-1812.

[31] Taber, K.S. (2003) Understanding Ionisation Energy: Physical, Chemical and Alternative Conceptions. Chemistry Education: Research and Practice, 4, 155-156. http://www.uoi.gr/cerp/2003_May/pdf/05Taber.pdf https://doi.org/10.1039/B3RP90010J

[32] Gross, J. (2011) Mass Spectrometry. 2nd Edition, Springer-Verlag, Berlin, Heidelberg, 26-29. https://doi.org/10.1007/978-3-642-10711-5

[33] McDonald, K.T. (2009) Radiation Pressure of a Monochromatic Plane Wave on a Flat Mirror. Joseph Henry Laboratories, Princeton University, Princeton, 1-6. 